\title{
Side to side coaptation - new technic in peripherial nerve surgery - preliminary report
}

\author{
P. Reichert' ${ }^{1}$, Z. Kiełbowicz ${ }^{2}$, J. Kuryszko ${ }^{3}$, A. Bocheńska ${ }^{4}$, \\ B. Puła ${ }^{5}$, R. Rutowski ${ }^{1}$
}

\footnotetext{
${ }^{1}$ Department of Traumatology, Clinic of Traumatology and Hand Surgery, Wroclaw Medical University, Borowska 213, 50-556, Wrocław, Poland

${ }^{2}$ Department of Surgery, Faculty of Veterinary Medicine, Wroclaw University of Environmental and Life Sciences, Pl. Grunwaldzki 51, 50-366, Wrocław, Poland

${ }^{3}$ Department of Animal Physiology and Biostructure, Faculty of Veterinary Medicine,

Wroclaw University of Environmental and Life Sciences, Norwida 31, 50-373, Wrocław, Poland

${ }^{4}$ Centre of Veterinary Medicine JU-UAK, Al. Mickiewicza 24/28, 30-059, Krakow

${ }^{5}$ Department of Histology and Embryology, Wroclaw Medical University,

Chałubińskiego 6a, 50-368, Wrocław, Poland
}

\begin{abstract}
This study presents and evaluates side-to-side nerve repair techniques for their ability to induce collateral nerve sprouting. The coaptation of the ventral branches of spinal nerves C5 and C6 to C7 through an incision epineurium was used to repair the nerve. The number of myelinated fiber axons and G-ratio was evaluated. Preliminary results indicate the possibility of using side to side coaptation in brachial plexus nerve surgery.
\end{abstract}

Key words: coaptation, roots avulsion, spinal nerve

\section{Introduction}

Avulsion of the roots of the brachial plexus are among the most severe peripheral nerve system lesions and until very recent years were treated as inoperable. In these cases end to end neurorrhaphy is impossible, because of the lack of the proximal stump. Therefore root avulsion injuries of the brachial plexus are usually treated by autologous nerve graft with end-to-end neurorrhaphy of a healthy donor nerve to the avulsed distal nerve stump. (Sananpachi et al.
2002). However, this approach has the drawback that it sacrifices a donor nerve and its target to provide reinnervation for the recipient nerve and target muscles (Schmidhammer et al. 2005). Recent reports prove that an alternative method may be use end-to-side coaptation (Viterbo et al. 1992). In this case the axons are derived from an uninjured brachial plexus component by collateral sprouting. This method is already used in the long branches of the brachial plexus. However, in the case of high brachial plexus injuries, at the level of the roots, the above

Correspondence to: P. Reichert, e-mail: pawelreichert74@gmail.com, tel.: 717343800 


\section{$\mathbf{A}$}

\section{(1)}

\begin{tabular}{l|l}
\hline $\mathbf{X}$ & $(2)$ \\
\hline
\end{tabular}

B

(1)
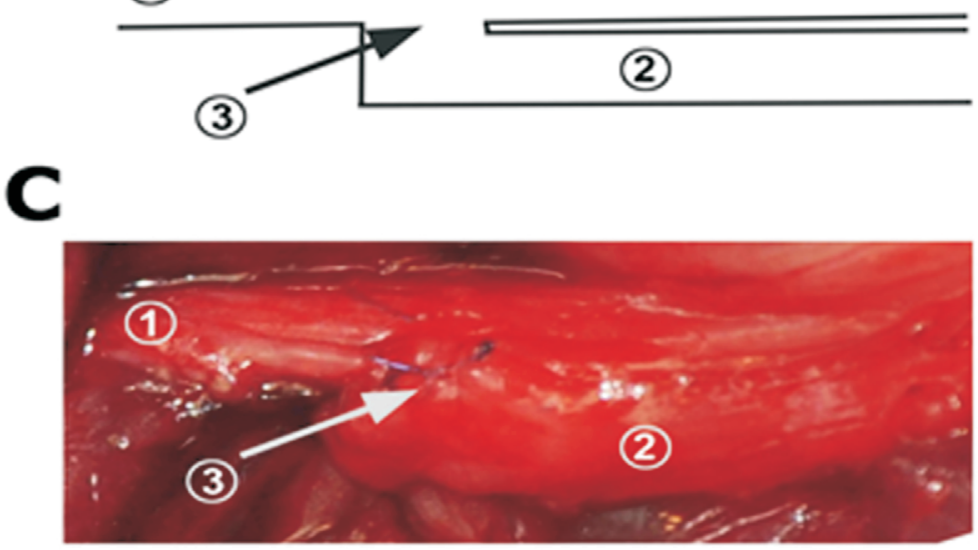

Fig. 1. Intraoperative picture and schematic illustration of surgical neurorrhaphy. A, B Schematic illustration. C Surgical photograph 1-uninjured nerve-donor nerve, 2-injured nerve-distal stump coapted nerve. 3. Side to side coaptation via epineural window. X-large defect injured nerve.

method, for anatomical reasons (short, wide trunk), is difficult to use. The purpose of this paper is to present and evaluate a new technique of side to side neurorrhaphy.

\section{Materials and Methods}

The experiments were approved by the Local Ethics Committee for animals at the University of Life Sciences in Wroclaw. In 6 New Zealand White rabbits side to side repairs on brachial plexus left limbs were made. All surgical procedures were performed with a lateral approach. The ventral branches of the spinal nerves C5 and C6 were avulsed from the spinal canal. Side-to-side repair was made via a $3-\mathrm{mm}$ window on right side ventral branches of spinal nerves C5 and C6, and the distal stump was sutured using 2 10-0 ethilon stitches via a 3-mm window on the left side ventral branches of spinal nerve C7 (Fig. 1a, 1b, 1c). The control group consisted of 6 right healthy limbs. The analysis was made on the basis of histological analysis of the number of myelinated fibers and a g-ratio (the ratio of the axon diameter to the diameter of the entire fiber) (Fig. 2).

\section{Results and Discussion}

Comparison of collected material from C5 and C6 $1 \mathrm{~cm}$ after coaptation to material from $\mathrm{C} 5$ and $\mathrm{C} 6$ on the same level in the control group shows that the number of myelinated axons was markedly reduced in side to side groups compared to the control group- $44 \%$. The number of axons myelin fiber density [fiber/mm2] was accordingly $143.22+/-4.4$ in the experimental and $342.19+/-2.643$ in control group. The G-ratio was respectively $0.64+/-0.08$ in the experimental group compared to $0.66+/-0.07$ in the control group, and the myelin sheath $[\mu \mathrm{m}] 1.54+/-0.66$ in the experimental group compared to $2.19+/-0.48$ in the control group. The results of the number of myelinated axons in the side to side coaptation group compared to the number of myelinated axons after end to side neurorrhaphy, is slightly lower than that known from the literature, where it is about $50 \%-60 \%$ of the 


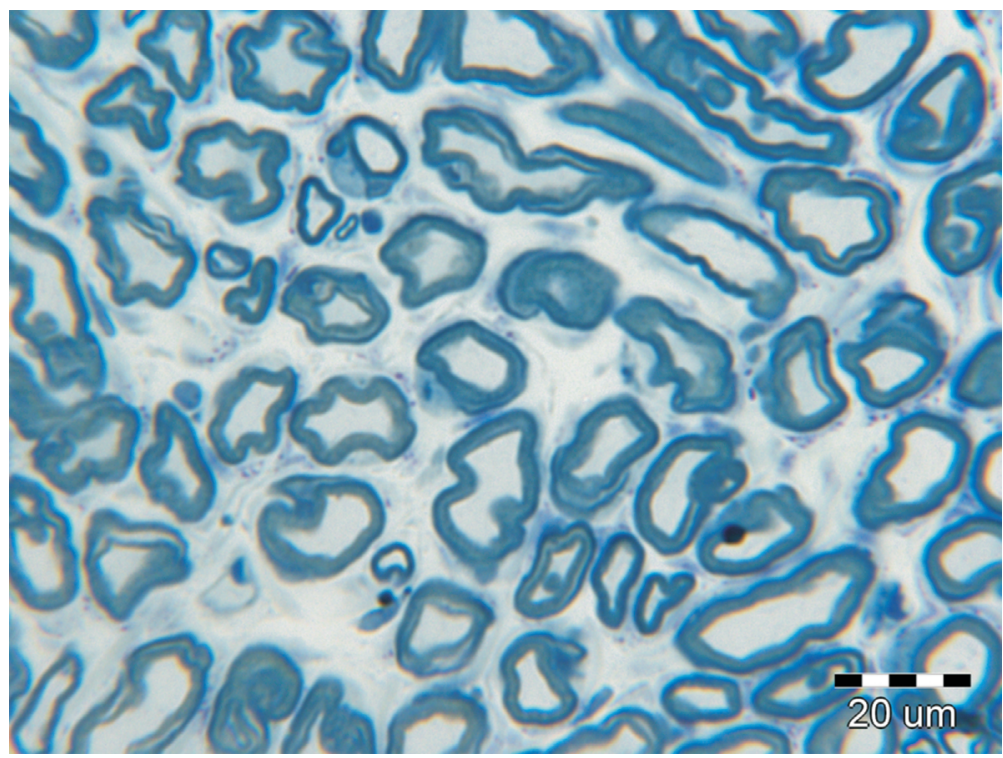

Fig. 2. Microscopic image of sampled nerve $1 \mathrm{~cm}$ from the coaptation. Toluidine blue staining.

original axons (Zhang 1999). On the other hand, the second essential parameter for collateral sprouting is correct value of G-ratio. As reported (Chomiak and $\mathrm{Hu}$ 2009), the G-ratio in peripheral nerve is more than 0.6. In our experimental group the result of G-ratio more than 0.6 indicates normal function of conduction in myelinated fibers. These results indicate a possibility of nerve conduction after side to side coaptation surgery. However, additional research is required, particularly retrograde labeling.

\section{Acknowledgements}

We would like to thank Bartosz Witkowski for providing medical writing service on behalf of Wroclaw Medical University.

\section{References}

Chomiak T, Hu B (2009) What is the optimal value of the g-ratio for myelinated fibers in the rat CNS? A theoretical approach. PLoS One 4: e7754.

Sanapanich K, Morrison WA, Messina A (2002) Physiologic and morphologic aspects of nerve regeneration after end-to-end or end-to-side coaptation in a rat model of brachial plexus injury. J Hand Surg Am 27: 133-142.

Schmidhammer R, Redl H, Hopf R, van der Nest DG, Millesi $\mathrm{H}$ (2005) End-to-side nerve graft repair based on synergistic peripheral terminal motor branches: investigation in a nonhuman primate model. Eur Surg 37: 308-316.

Viterbo F, Trindade JC, Hoshino K, Mazzoni Neto A (1992) Latero-terminal neurorrhaphy without removal of the epineural sheath. Experimental study in rats. Rev Paul Med 110: 267-275.

Zhang Z, Soucacos PN, Bo J, Beris AE (1999) Evaluation of collateral sprouting after end-to-side nerve coaptation using a fluorescent double-labeling technique. Microsurgery 19: 281-286. 